\section{Integrated mRNA and Small RNA Sequencing for Analyzing Tea Leaf Spot Pathogen Lasiodiplodia theobromae, Under In Vitro Conditions and the Course of Infection}

\author{
Silong Jiang, ${ }^{1,2}$ Qiaoxiu Yin, ${ }^{1}$ Dongxue Li, ${ }^{1}$ Xian Wu,,${ }^{1}$ Yong Wang, ${ }^{2}$ Delu Wang,,${ }^{3, \dagger}$ and Zhuo Chen ${ }^{1, \dagger}$ \\ ${ }^{1}$ Key Laboratory of Green Pesticide and Agricultural Bioengineering, Ministry of Education, Guizhou \\ University, Guiyang, Guizhou 550025, China \\ ${ }^{2}$ Agricultural College, Guizhou University, Guiyang, Guizhou 550025, China \\ ${ }^{3}$ College of Forestry, Guizhou University, Guiyang, Guizhou 550025, China
}

\begin{abstract}
Lasiodiplodia theobromae is a phytopathogenic fungus, which can cause many different diseases on different crops. The pathogen can cause leaf spot on tea plants (Camellia sinensis), which negatively affects the productivity and quality of tea leaves in tea plantations in Guizhou Province, China. Although the genome sequence of $L$. theobromae has been published, no data on the transcriptome or small RNA sequences of $L$. theobromae under in vitro conditions and the course of infection of tea leaf are available. Here, we report the high-quality transcriptome and small RNA sequences of $L$. theobromae in vitro conditions and the course of infection of tea leaf using the platform of Illumina HiSeq. This comprehensive expression profiling of the fungal pathogen will provide a valuable resource for future research on trait-specific genes of the pathogen, host-pathogen interactions, and disease resistance in the host.
\end{abstract}

\section{Transcriptome and Small RNA Sequence Announcement}

The fungal family Botryosphaeriaceae (phylum Ascomycota) includes saprobes, endophytes, and phytopathogens (Chethana et al. 2016; Morales-Cruz et al. 2015; Phillips et al. 2013; Yan et al. 2013). Many members of this family can enter the host tissues via wounds, lenticels, or stomata (Morales-Cruz et al. 2015; Yan et al. 2013), and live as endophytes, changing to act like pathogens when the host is stressed (Chethana et al. 2016; PaolinelliAlfonso et al. 2016). At present, the family Botryosphaeriaceae is divided into 23 genera, of which Botryosphaeria Ces. \& De Not., Diplodia Fr., Lasiodiplodia Ellis \& Everh., and Neofusicoccum Crous, Slippers \& A. J. L. Phillips are considered to be the genera containing the most virulent phytopathogens (Phillips et al. 2013; Yan et al. 2013). L. theobromae Pat. Griffon \& Maubl. is the anamorph of Botryosphaeria rhodina (Berk. \& Curtis) Arx (Punithalingam 1980).

This fungus can cause diseases in many unrelated plant species throughout the tropical and subtropical regions, such as canker and dieback of grapevine (Rodríguez-Gálvez et al. 2015), branch canker of olive (Pérez et al. 2017), branch dieback of cashew (Cardoso et al. 2002), fruit spot of eggplant (Woodward et al. 2005), fruit rot of pomelo (Luo et al. 2011), postharvest fruit rot in avocado (Garibaldi et al. 2012), gummosis or inflorescence blight of mango trees (Li et al. 2013; Serrato-Diaz et al. 2013), inflorescence blight and fruit rot of longan (Serrato-Diaz et al. 2014), leaf spot of tea oil Camellia (Camellia oleifera) (Zhu et al. 2014), collar rot of peanut (Phipps and Porter 1998), and strawberry dieback (Yildiz et al. 2014). Recently, our

\footnotetext{
${ }^{\dagger}$ Corresponding authors: Z Chen; gychenzhuo@aliyun.com, and D. Wang; fc.dlwang@gzu.edu.cn

*The $e$-Xtra logo stands for "electronic extra" and indicates that supplementary tables are published online.
}

The author(s) declare no conflict of interest.

Accepted for publication 8 December 2020.

\section{Funding}

The project was supported by National Key Research Development Program of China (2017YFD0200308) and its Postsubsidy project (2018-5262), the National Natural Science Foundation of China (No. 31860515 and 21977023), Program of Introducing Talents to Chinese Universities (111 Program, D20023), and the China Agriculture Research System (CARS-23-D09).

\section{Keywords}

bioinformatics, fungal pathogens, genomics 
research group reported that $L$. theobromae caused tea leaf spot on tea bushes in plantations, significantly decreasing the production and quality of tea (Li et al. 2019; Ren et al. 2019).

Single conidia of $L$. theobromae isolate GZHS-2017-010 were transferred to potato dextrose agar and then incubated for 24 days. The conidial suspensions were prepared from the colony and then transferred to potato dextrose broth $(\mathrm{PDB})$ at $33^{\circ} \mathrm{C}$ and $200 \mathrm{rpm}$ in the dark for $32 \mathrm{~h}$. The mycelia in PDB were obtained and ground into a fine powder in liquid nitrogen for RNA extractions, using the TRIzol Plus RNA Purification Kit (Invitrogen, Carlsbad, CA, U.S.A.). The conidial suspensions of $L$. theobromae isolate GZHS-2017-010 $\left(10^{6}\right.$ conidia/ml, 20 to $\left.30 \mu \mathrm{l}\right)$ were inoculated onto wounded tea leaves. After incubation for $48 \mathrm{~h}$, a lesion had formed on the leaf. Total RNA of tea leaf was extracted using TRIzol Plus RNA Purification Kit (Invitrogen), following the manufacturer's procedure. A cDNA library was prepared using the mRNASeq sample preparation kit (Illumina) and sequenced on an Illumina Hiseq 4000 by Lc-Bio Technologies Co., Ltd. (Hangzhou, China) following the manufacturer's recommended protocol. Cutadapt (https://github.com/marcelm/cutadapt/) was used to remove the reads that contained adapter contamination, low-quality bases (the ratio of false recognition to a specific read is above $1 \%$ during the bases calling) and undetermined bases (Martin 2011). Then, sequence quality was verified using FastQC (http://www.bioinformatics.babraham.ac.uk/projects/fastqc/), including the Q20 and Q30 indexes and the GC content of the clean data. All downstream analyses were based on clean data of high quality. Sequencing reads were aligned to the reference genome (VCHE01, BioProject PRJNA449280) using HISAT2 package (version 2.0) (http://www.ccb.jhu.edu/software/hisat/index.shtml) (Kim et al. 2015). StringTie software (https://ccb.jhu.edu/software/stringtie/) and edgeR software were used to estimate the expression levels of all transcripts (Pertea et al. 2015; Robinson et al. 2010). The differentially expressed genes (DEGs) were selected by $R$ package with $\log _{2}$ (fold change) values of $\geq 1$ or $\log _{2}$ (fold change) values of $\leq-1$ and with statistical significance of $P<0.05$. DEGs were annotated using DIAMOND software (version 0.7.12) (http://ab.inf.uni-tuebingen.de/software/ diamond) (Buchfink et al. 2015) and gene ontology (GO) (http://geneontology.org) (version 2016.04) and Kyoto Encyclopedia of Genes and Genomes (KEGG) (https://www.kegg.jp/kegg) (version 2016.05) databases.

Libraries of small RNAs were prepared using TruSeq Small RNA Sample Prep Kits (Illumina) from the RNA extracted from both the pure mycelia and infected plant material, and sequenced by Illumina HiSeq 2500 (Illumina), with single-end sequencing, and a sequencing read length of $1 \times 50 \mathrm{bp}$. Raw reads were analyzed using ACGT101-miR (LC Sciences, Houston, TX, U.S.A.) to remove adapter dimers, junk, low-complexity, common RNA families (ribosomal RNA, transfer RNA, small nuclear RNA, and small nucleolar RNA), and repeats. Reads with lengths of 18 to 25 nucleotides were retained, and unique sequences with lengths of 18 to 25 nucleotides were mapped to specific species precursors in miRBase 22.0 (ftp://mirbase.org/pub/mirbase/CURRENT/) by BLAST search, to identify known MicroRNAs (miRNAs) and novel 3p- and 5p-derived miRNAs. The rest of the sequences were aligned through the databases of mRNA, RFam (http://rfam.janelia.org), and Repbase (https://www.girinst.org/ repbase), and filtered to form valid data. miRNAs were aligned with the precursor and genome and then identified. Target genes of individual miRNAs were predicted using TargetFinder (http://targetfinder.org/) (Dai and Zhao 2011), and their enrichment analysis was conducted using GO (http://geneontology.org/) and KEGG (https://www.genome.jp/kegg/).

The numbers of genes and transcripts of $L$. theobromae isolate GZHS-2017-010 for two treatments (pathogen grown in PDB and pathogen grown in tea leaves) were 12,785 and 12,896 . The numbers of DEGs of $L$. theobromae isolate GZHS-2017-010 were 2,251 and 1,664 for up-regulation and down-regulation, respectively. DEGs of $L$. theobromae were annotated using GO, with the numbers of $S$ genes (gene with significantly differential expression was annotated in special GO) being significantly enriched with respect to the oxidation-reduction process, transmembrane transport, and metabolic process at the level of biological process (BP) being 304, 181, and 118, respectively. At the level of cellular component (CC) aspect, nucleus, cytosol, and integral component of membrane were significantly enriched, with the numbers of $S$ genes being 302,281 , and 213 , respectively. At the level of molecular function (MF) aspect, oxidoreductase activity, catalytic activity, and zinc ion binding were significantly enriched, with the numbers of $S$ genes being 185,170 , and 130, respectively. The pathways of glycine, serine, and threonine metabolism, tryptophan metabolism, and tyrosine metabolism were significantly enriched in the inoculated leaves, relative to the control leaves, with the numbers of $S$ genes in each of these three terms being 64,60 , and 51 , respectively (Table 1 ). 
Table 1. Transcriptome and small RNA sequences of Lasiodiplodia theobromae isolate GZHS-2017-010 and tea (Camellia sinensis), under in vitro conditions and the course of infection

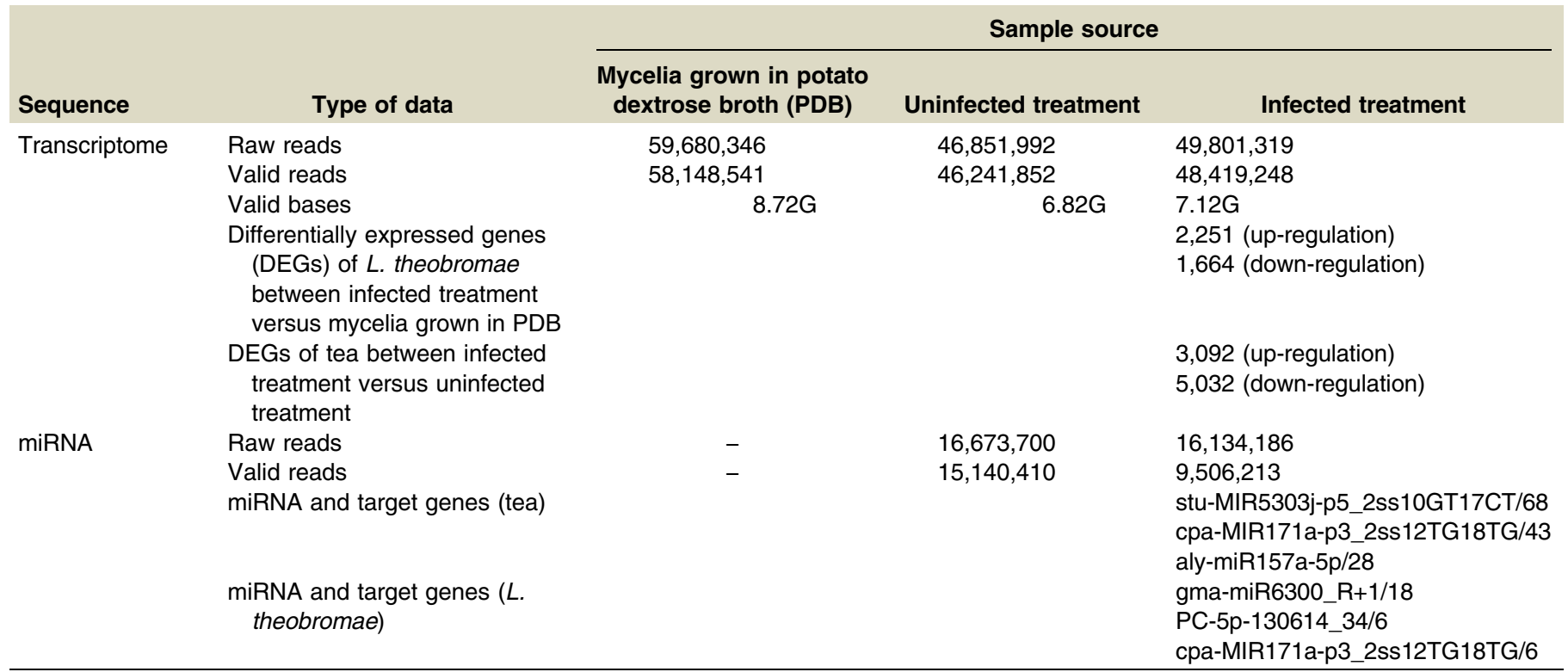

miRNAs were divided into gp2, gp3, and gp4, and the target gene of miRNAs were identified and predicted. stu-MIR5303j-p5_2ss10GT17CT, cpa-MIR171a-p3_2ss12TG18TG, aly-miR157a-5p, gma-miR166a-3p, and gma-MIR167c-p3_2ss14TA20AG targeted the most genes of tea, with the number of the target genes being $68,43,28,23$, and 21 , respectively. The target genes were significantly enriched with respect to regulation of transcription, DNAtemplated transcription, DNA-templated BP, cell differentiation, and meristem maintenance at the level of BP, with the numbers of $S$ genes being $41,28,25,19$, and 18, respectively. At the level of CC, nucleus, cytoplasm, plasma membrane, chloroplast, and integral component of membrane were significantly enriched, with the number of $S$ genes being 111, 37, 35, 28, and 27 , respectively. At the level of MF, DNA-binding transcription factor activity, DNA binding, protein binding, transaminase activity, and metal ion binding were significantly enriched, with the number of $S$ genes being 36, 28, 23, 19, and 19, respectively. The target genes were significantly enriched in the pathways of amino sugar and nucleotide sugar metabolism, plant hormone signal transduction, and zeatin biosynthesis, with the number of $S$ genes being 21,12 , and 6, respectively. The numbers of DEGs of tea were 3,092 and 5,032 for up-regulation and down-regulation, respectively. DEGs of tea were annotated using GO, with the numbers of $S$ genes being significantly enriched with respect to the ATP binding, metal ion binding, and protein kinase activity at the level of MF being 404, 178, and 143, respectively (Table 1).

gma-miR6300_R+1, PC-5p-130614_34, and cpa-MIR171a-p3_2ss12TG18TG can target more genes of $L$. theobromae, with the number of target genes being 18, 6 , and 6 , respectively. The target genes were significantly enriched with respect to pathogenesis, regulation of transcription, and DNA-templated transmembrane transport at the level of BP, with the number of S genes being 3, 3, and 3, respectively. The target genes were significantly enriched in the pathways of ubiquitin-mediated proteolysis, RNA degradation, and starch and sucrose metabolism, with the number of $S$ genes being 2,2 , and 2 , respectively (Table 1).

The transcriptome and small RNA sequences of $L$. theobromae, grown in PDB or expressed at different stages during infection of tea leaves, will provide an important resource for researchers studying the pathogenic mechanism and the disease resistance response in the $L$. theobromae-C. sinensis combination. The sequence of transcriptome and small RNA sequences of $L$. theobromae have been deposited in GenBank under sequence read archive (SRA) accession PRJNA540200 (accessions SRX5762638, SRX5762639, and SRX5762640) and SRA accession number PRJNA637660 for the transcriptome data, and under SRA accession number PRJNA558429 for the miRNA data. In addition, bioinformatics analysis of transcriptome and microRNA are presented in Supplementary Table S1. L. theobromae isolate 


\section{Literature Cited}

Buchfink, B., Xie, C., and Huson, D. H. 2015. Fast and sensitive protein alignment using diamond. Nat. Methods 12:59-60.

Cardoso, J. E., Vidal, J. C., Santos, A. A. D., Freire, F. C. O., and Viana, F. M. P. 2002. First report of black branch dieback of cashew caused by Lasiodiplodia theobromae in brazil. Plant Dis. 86:558.

Chethana, K. W. T., Li, X. H., Zhang, W., Hyde, K. D., and Yan, J. Y. 2016. Trail decryption of molecular research on Botryosphaeriaceae in woody plants. Phytopathol. Mediterr. 55:147-171.

Dai, X., and Zhao, P. X. 2011. psRNATarget: A plant small RNA target analysis server. Nucleic Acids Res.: W155-W159.

Garibaldi, A., Bertetti, D., Amatulli, M. T., Cardinale, J., and Gullino, M. L. 2012. First report of postharvest fruit rot in avocado (Persea americana) caused by Lasiodiplodia theobromae in Italy. Plant Dis. 96:460.

Kim, D., Langmead, B., and Salzberg, S. L. 2015. HISAT: A fast spliced aligner with low memory requirements. Nat. Methods 12:357-360.

Li, D. X., Bao, X. T., Ren, Y. F., Wang, Y., Song, B. A., and Chen, Z. 2019. First report of Lasiodiplodia theobromae causing leaf spot on tea plant in Guizhou Province of China. Plant Dis. 103:374.

Li, Q. L., Guo, T. X., Pan, Z. B., Huang, S. P., Mo, J. Y., Ning, P., and Hsiang, T. 2013. An outbreak of gummosis of mango trees caused by Lasiodiplodia theobromae in Guangxi, South China. Plant Dis. 97:690.

Luo, M., Dong, Z. Y., Bin, S. Y., and Lin, J. T. 2011. First report of fruit rot disease on pomelo caused by Lasiodiplodia theobromae in China. Plant Dis. 95:1190.

Martin, M. 2011. Cutadapt removes adapter sequences from high-throughput sequencing reads. EMBnet. J. 17:10-12.

Morales-Cruz, A., Amrine, K. C. H., Blanco-Ulate, B., Lawrence, D. P., Travadon, R., Rolshausen, P. E., Baumgartner, K., and Cantu, D. 2015. Distinctive expansion of gene families associated with plant cell wall degradation, secondary metabolism, and nutrient uptake in the genomes of grapevine trunk pathogens. BMC Genomics 16:469.

Paolinelli-Alfonso, M., Villalobos-Escobedo, J. M., Rolshausen, P., Herrera-Estrella, A., Galindo-Sánchez, C., López-Hernández, J. F., and Hernandez-Martinez, R. 2016. Global transcriptional analysis suggests Lasiodiplodia theobromae pathogenicity factors involved in modulation of grapevine defensive response. BMC Genomics 17:615.

Pérez, B. A., Niz, J., Salvador, R., Berretta, M., Roca, M. E. M., Pesce, M. V., Matías, A. C., González De Urreta, M., Paccioretti, M., and Otero, M. L. 2017. First report of Lasiodiplodia theobromae causing branch canker on 'Manzanilla' olive in Northwestern Argentina. Plant Dis. 102:677.
Pertea, M., Pertea, G. M., Antonescu, C. M., Chang, T. C., Mendell, J. T., and Salzberg, S. L. 2015. StringTie enables improved reconstruction of a transcriptome from RNAseq reads. Nat. Biotechnol. 33:290-295.

Phillips, A. J. L., Alves, A., Abdollahzadeh, J., Slippers, B., Wingfield, M. J., Groenewald, J. Z., and Crous, P. W. 2013. The Botryosphaeriaceae: Genera and species known from culture. Stud. Mycol. 76:51-167.

Phipps, P. M., and Porter, D. M. 1998. Collar rot of peanut caused by Lasiodiplodia theobromae. Plant Dis. 82:1205-1209.

Punithalingam, E. 1980. Plant diseases attributed to Botryodiplodia theobromae Pat. Bibl. Mycol. 71:1-123.

Ren, Y. F., Li, D. X., Zhao, X. Z., Wang, Y., Bao, X. T., Wang, X., Wu, X., Wang, D. L., Song, B. A., and Chen, Z. 2019. Whole genome sequences of the tea leaf spot pathogen Didymella segeticola. Phytopathology 109:1676-1678.

Robinson, M. D., McCarthy, D. J., and Smyth, G. K. 2010. edgeR: A bioconductor package for differential expression analysis of digital gene expression data. Bioinformatics 26:139-140.

Rodríguez-Gálvez, E., Maldonado, E., and Alves, A. 2015. Identification and pathogenicity of Lasiodiplodia theobromae causing dieback of table grapes in Peru. Eur. J. Plant Pathol. 141:477-489.

Serrato-Diaz, L. M., Perez-Cuevas, M., Rivera-Vargas, L. I., and French-Monar, R. D. 2013. First report of Lasiodiplodia theobromae causing inflorescence blight of mango. Plant Dis. 97:1380.

Serrato-Diaz, L. M., Rivera-Vargas, L. I., Goenaga, R., and French-Monar, R. D. 2014. First report of Lasiodiplodia theobromae causing inflorescence blight and fruit rot of longan (Dimocarpus longan L.) in Puerto Rico. Plant Dis. 98:279.

Woodward, J. E., Langston, D. B., Brock, J. H., Kemerait, C. J., Brenneman, T. B., and Beard, G. H. 2005. First demonstration of Koch's postulates for Lasiodiplodia theobromae fruit spot on eggplant (Solanum melongena). Plant Dis. 89:687.

Yan, J. Y., Xie, Y., Zhang, W., Wang, Y., Liu, J. K., Hyde, K. D., Seem, R. C., Zhang, G. Z., Wang, Z. Y., Yao, S. W., Bai, X. J., Dissanayake, A. J., Peng, Y. L., and Li, X. H. 2013. Species of Botryosphaeriaceae involved in grapevine dieback in China. Fungal Divers. 61:221-236.

Yildiz, A., Benlioglu, K., and Benlioglu, H. S. 2014. First report of strawberry dieback caused by Lasiodiplodia theobromae. Plant Dis. 98:1579.

Zhu, H., Niu, X. Q., Song, W. W., Yu, F. Y., Tang, Q. H., Qin, W. Q., and Chen, L. Q. 2014. First report of leaf spot of tea oil camellia (Camellia oleifera) caused by Lasiodiplodia theobromae in China. Plant Dis. 98:1427. 